\title{
Adaptive optics without borders: performance evaluation in the infinite aperture limit
}

\section{Brent L. Ellerbroek}

Brent L. Ellerbroek, "Adaptive optics without borders: performance evaluation in the infinite aperture limit," Proc. SPIE 5490, Advancements in Adaptive Optics, (25 October 2004); doi: 10.1117/12.548337

EDent: SPIE Astronomical Telescopes + Instrumentation, 2004, Glasgow, United Kingdom 


\title{
Adaptive optics without borders: performance evaluation in the infinite aperture limit
}

\author{
Brent L. Ellerbroek \\ AURA New Initiatives Office, 950 N. Cherry Ave., Tucson AZ, 85719
}

\begin{abstract}
The limit case of an infinite aperture adaptive optics (AO) system eliminates the modeling complications associated with aperture edge effects, and thereby enables the application of simplified methods for system performance evaluation in the spatial frequency domain. We review prior work in this field and describe a new approach that enables a wider range of error sources and $\mathrm{AO}$ options to be evaluated with a reduced number of approximations. These errors and AO options include: Fitting error and spatial aliasing for a Shack-Hartmann wavefront sensor (WFS) and one particular deformable mirror influence function; WFS noise; servo lag for a continuous temporal filter function; anisoplanatism in either a single evaluation direction or averaged over an extended field of view; piston removal within a finite aperture; minimum variance and modal wavefront reconstruction algorithms; and multi-conjugate AO. Laser guidestars, however, are excluded. A wide range of classical results for the independent effects of individual error sources can be immediately derived from this integrated model. Performance estimates for more complex problems involving the full range of first-order AO error sources are in good agreement with the results produced by more detailed Monte Carlo simulations.
\end{abstract}

Keywords: Adaptive optics modeling and simulation

\section{INTRODUCTION}

Adaptive optical (AO) instrumentation continues to play a highly prominent role in the emerging plans for the next generation of ground based astronomical telescopes. ${ }^{1,2}$ It is likely that the range of AO concepts proposed for these telescopes will continue to grow in terms of variety, complexity, and dimensionality. In the last several years, good to excellent progress has been made in developing efficient algorithms and simulations for detailed modeling of these concepts. ${ }^{3-7}$ However, even the most efficient algorithms and fastest available processors are severely challenged by $\mathrm{AO}$ systems with $10^{4}$ to $10^{5}$ degrees of freedom, and each individual simulation may take many hours, or even days, of computer time. Exploring a broad range of potential system options is not practical with this approach, and there remains a need for simpler methods that are fast, general, and still reasonably accurate.

A wide range of useful formulas for efficiently (albeit approximately) evaluating the impact of the individual error sources in an AO system have already been developed using spatial frequency domain techniques. Examples include models for anisoplanatism, ${ }^{8}$ servo lag, ${ }^{9,10}$ wavefront sensor (WFS) spatial aliasing, ${ }^{11}$ and tomographic wavefront reconstruction in multi-conjugate AO (MCAO). ${ }^{12,13}$ These results exploit the fact that the fundamental optical and control processes to be considered-wavefront propagation, sensing, reconstruction, and correction-are well modeled as spatial filtering operations. Many important AO error sources may consequently be evaluated singly in terms of simple scaling laws or somewhat more complex functions of a single parameter. To date, however, less progress has been made in developing methods for efficiently evaluating the combined, and partially correlated, impact of multiple AO error sources. The usual approach is to incoherently sum or convolve the contributions of the individual terms, ${ }^{14,15}$ which generally yields a (possibly pessimistic) lower bound on performance.

In this paper, we describe a more integrated approach to spatial frequency domain modeling that correctly captures many of the interactions and correlations between the most fundamental error sources in an AO system. Our starting point is a standard linear systems model that treats the optical and control processes in AO as linear operators acting on abstract vector spaces of turbulence screens, phase profiles, WFS measurements, and DM

Further author information: E-mail: brent@noao.edu, Telephone: 1 (520) 318-8589 
actuator commands. Virtually all formulas for classical wavefront reconstruction algorithms and their expected performance are expressed in terms of these operators and the second-order statistics of turbulence screens and WFS measurement noise. All of these quantities are diagonal with respect to spatial frequency in the Fourier domain, and it follows that reconstruction algorithms may be derived and evaluated one Fourier component at a time. With the aid of this decoupling, even the very high order AO systems presently under study for future extremely large telescopes may be optimized and evaluated in a matter of minutes on a desktop personal computer.

This simplicity and efficiency comes at the expense of neglecting the boundary conditions and aperture edge effects that cannot be represented via shift-invariant spatial filters. On account of this approximation, this technique should be viewed primarily as a method for obtaining fast and reasonably accurate performance estimates during (for example) the initial stages of developing system requirements. Without treating aperture edge effects, it cannot be used to generate the reconstruction matrix for an actual AO system. Considered as an analysis tool, however, it models many of the phenomena and options that define the landscape of classical wavefront reconstructor theory. These features include: Integrated treatment of five fundamental AO error sources (DM fitting error, WFS spatial aliasing, additive WFS measurement noise, anisoplanatism, and servo lag); multi-guidestar $\mathrm{AO}$ and $\mathrm{MCAO}$; least squares, minimum variance, and closed-loop minimum variance wavefront reconstruction; zonal and modal wavefront control; and models for the AO-compensated point spread function (PSF) as a function of wavelength and evaluation direction. All of the standard formulations for these concepts are transferred faithfully to the spatial frequency domain, apart from neglecting aperture edge effects.

The remainder of this paper sketches the elements of this method and presents a range of sample numerical results. Section 2 reviews some of the basic elements of the standard AO linear systems model that is the starting point for this work. Section 3 summarizes the usual representations for some of the operators and statistical processes comprising this model, and confirms that they may all be expressed as spatial filters or $\delta$-correlated power spectra. Section 4 validates one of the analytical results obtained using the new model against classical formulas for the effect of WFS measurement noise in a conventional, single-conjugate AO system. Section 5 applies the model to study the interaction of multiple error sources in an 8-meter class MCAO system. The agreement with both classical scaling laws and detailed simulations ranges from good to exact in both of these test cases.

\section{LINEAR SYSTEMS MODELS}

The present work is based upon a set of standard, well known results for describing and optimizing AO performance using classical linear systems techniques. ${ }^{16-20}$ They are applicable to AO architectures using either single or multiple wavefront sensors (WFS's) and deformable mirrors (DM's) for turbulence compensation across either a narrow or wide field-of-view (FOV). It is convenient to group these results into three categories of increasing sophistication related to (i) fitting DM actuator commands to a known turbulence profile, (ii) reconstructing turbulence profiles from noisy and incomplete WFS measurements, and (iii) evaluating the dynamical response of a classical, closed-loop AO system to time-varying turbulence and WFS measurement noise. Space limitations prevent us from presenting all of this material, but the following paragraphs review wavefront fitting in detail and provide a very brief summary of results on wavefront reconstruction and control.

\subsection{Wavefront Fitting}

Given a linear (geometrical optics) model for wavefront propagation, the residual phase profile $\phi$ obtained by an AO system when a set of DM actuator commands $a$ are applied to correct for the turbulence profile $x$ can be described by an equation of the form

$$
\phi=\phi(a)=H_{x} x-H_{a} a,
$$

where the matrices $H_{x}$ and $H_{a}$ are the influence matrices that describe the the impact of $x$ and $a$ upon the output wavefront $\phi(a)$. The quantities $x, a$, and $\phi$ are vectors described in terms of arbitrary basis functions, and in the general case represent (i) multiple 2-dimensional turbulence screens in a distributed, 3-dimensional atmosphere, (ii) actuator commands for multiple DM's that are optically conjugate to distinct ranges in the atmosphere, and (iii) multiple 2-dimensional phase profiles propagated from distinct science objects distributed over an extended FOV. 
The aperture- and field-averaged variance of $\phi(a)$ is a standard metric for the residual phase error. The general form of $\sigma^{2}(a)=\operatorname{var}[\phi(a)]$ is the expression

$$
\sigma^{2}(a)=\phi^{T}(a) W_{\phi} \phi(a),
$$

where the symmetric, semi-positive-definite weighting matrix $W_{\phi}$ is determined by (i) any cross-coupling between the components of $\phi(a)$ due to the choice of basis set, and (ii) for a wide FOV AO system, the relative importance attached to the different science objects.

Evaluating the variance $\sigma^{2}(a)$ for either the optimal choice of $a$ or various suboptimal choices is a recurring problem in adaptive optics. ¿From the above equations it follows that $\sigma^{2}(a)$ is quadratic in $a$, so the optimum solution may be determined using standard least-squares methods. The result is described by the equations

$$
\begin{aligned}
a_{*} & \equiv \arg \min _{a} \sigma^{2}(a)=W_{a}^{-1} C_{a x} x, \\
\sigma^{2}\left(a_{*}\right) & =x^{T}\left[W_{x}-C_{a x}^{T} W_{a}^{-1} C_{a x}\right] x, \\
\sigma^{2}(a) & =\sigma^{2}\left(a_{*}\right)+\left(a_{*}-a\right)^{T} W_{a}\left(a_{*}-a\right),
\end{aligned}
$$

where we have introduced the variables $W_{a}=H_{a}^{T} W_{\phi} H_{a}, C_{a x}=H_{a}^{T} W_{\phi} H_{x}$, and $W_{x}=H_{x}^{T} W_{\phi} H_{x}$ to simplify notation. The turbulence profile $x$ is a random quantity, so the time-averaged value of $\sigma^{2}(a)$ is also of interest for many applications. The usual notation, $\langle\ldots\rangle$, will be used to denote ensemble averages over random quantities including turbulence profiles, WFS measurement noise, and (in some cases) the variability of the wind. The expected values of $\sigma^{2}\left(a_{*}\right)$ and $\sigma^{2}(a)$ can be determined from Eq.'s (4) and (5) with the result ${ }^{16}$

$$
\begin{aligned}
\left\langle\sigma^{2}\left(a_{*}\right)\right\rangle & =\operatorname{tr}\left[W_{x}\left\langle x x^{T}\right\rangle\right]-\operatorname{tr}\left[W_{a}^{-1} C_{a x}\left\langle x x^{T}\right\rangle C_{a x}^{T}\right], \\
\left\langle\sigma^{2}(a)\right\rangle & =\left\langle\sigma^{2}\left(a_{*}\right)\right\rangle+\operatorname{tr}\left[W_{a}\left\langle\left(a_{*}-a\right)\left(a_{*}-a\right)^{T}\right\rangle\right] .
\end{aligned}
$$

Here $\operatorname{tr}(M)$ denotes the trace (or sum of the diagonal elements) of a square matrix $M$.

\subsection{Wavefront Estimation}

The best-fit actuator command vector $a_{*}$ is generally not known a priori, but instead must be reconstructed (i.e., estimated) from a noisy and incomplete WFS measurement vector. We consider first the simpler (and generally unrealistic) case where the measurement depends upon the turbulence disturbance $x$ but not the DM actuator command vector $a$. Assuming geometrical optics and an ideal, linear WFS, this so-called "open-loop" measurement $s_{0}$ is described by the formula

$$
s_{0}=G_{x} x+n,
$$

where $G_{x}$ is the turbulence-to-WFS influence matrix and $n$ is an additive noise term that is assumed to be statistically independent of $x$. In analogy with $\phi$, the measurement $s_{0}$ may include components recorded by several different sensors observing distinct guidestars distributed over the FOV. We will assume that reconstruction algorithm is a linear operator, and can therefore be represented by a matrix multiply of the form

$$
a=R s_{0} .
$$

The matrix $R$ should be selected so that the reconstructed set of DM commands $a$ is a good approximation to the best-fit value $a_{*}$. This criteria may be quantified in terms of $\left\langle\sigma^{2}\left(R s_{0}\right)\right\rangle$, the expected variance of the residual phase error. Eq. (7) may be used to compute this quantity, once the covariance matrix $\left\langle\left(a_{*}-a\right)\left(a_{*}-a\right)^{T}\right\rangle=$ $\left\langle\left(a_{*}-R s_{0}\right)\left(a_{*}-R s_{0}\right)^{T}\right\rangle$ has been determined. Using the commutative properties of $\langle\ldots\rangle$ together with Eq.'s (8) and (3), we obtain

$$
\left\langle\left(a_{*}-R s_{0}\right)\left(a_{*}-R s_{0}\right)^{T}\right\rangle=\left\langle a_{*} a_{*}^{T}\right\rangle-R\left\langle s_{0} a_{*}^{T}\right\rangle-\left\langle a_{*} s_{0}^{T}\right\rangle R^{T}+R\left\langle s_{0} s_{0}^{T}\right\rangle R^{T},
$$

where the covariance matrices involving $a_{*}$ and $s_{0}$ may be expressed in terms of the statistics of $x$ and $n$ using Eq.'s (3) and (8). 
It is frequently of interest to optimize the reconstruction algorithm, if only to provide a bound on the performance of any possible approach. The so-called "minimum variance reconstructor" is given by the expression

$$
R_{*} \equiv \arg \min _{R}\left\langle\sigma^{2}\left(R s_{0}\right)\right\rangle=\left\langle a_{*} s_{0}^{T}\right\rangle\left\langle s_{0} s_{0}^{T}\right\rangle^{-1}
$$

It is possible to proceed further and develop concise formulas for (i) the minimized phase variance $\left\langle\sigma^{2}\left(R_{*} s_{0}\right)\right\rangle$ and (ii) the incremental error incurred by using a suboptimal value of $R$ instead of $R_{*}$.

\subsection{Wavefront Control}

The formulation of the wavefront reconstruction problem outlined above is oversimplified for several important reasons. The large majority of operational and planned AO systems employ wavefront sensors with limited linear dynamic ranges, and therefore place the WFS following the deformable mirror so that only the (smaller) residual wavefront errors are sensed. The output of the wavefront reconstruction algorithm computed at each cycle of the control loop is used to update the prior set of DM actuator commands, with a temporal filter applied to reduce the effects of measurement noise and provide more stable control. The dynamics of the system may be represented by the formulas

$$
\begin{aligned}
& s(t)=s_{0}(t)-G_{a} a(t) \\
& a(t)=\int_{0}^{\infty} d \tau f(\tau)[R s(t-\tau)] .
\end{aligned}
$$

Here $t$ and $\tau$ are time variables, $s(t)$ is the closed-loop WFS measurement after correction by the DM actuator command $a(t), G_{a}$ is the DM-to-WFS influence matrix, and $f(\tau)$ is the (scalar-valued) function used to filter the output of the wavefront reconstruction operator before it is applied to the DM. Eq. (12) and (13) describe a classical control architecture as implemented in many currently operational AO systems, as distinct from some of the more innovative approaches now being studied via analysis and simulation.

The explicit dependence of $a(t)$ upon $s(t)$ may be determined using Fourier transform techniques. In the general case, the relationship is described by a matrix-valued filter, making it computationally difficult to evaluate and (especially) to optimize the performance of the AO control loop. These difficulties may be avoided by restricting attention to values of $R$ that satisfy the condition $R G_{a}=I$, i.e., wavefront reconstructors that are left pseudo-inverses of the DM-to-WFS influence matrix. In this special case the temporal dynamics of the AO system are defined by the expression

$$
a(t)=R s_{f}(t)=R\left[G_{x} x_{f}(t)+n_{f}(t)\right],
$$

where $s_{f}(t), x_{f}(t)$, and $n_{f}(t)$ are temporally filtered versions of the quatities $s_{0}(t), x(t)$, and $n(t)$ that have been convolved with the closed-loop impulse response function $f_{I R}(t)$ corresponding to the servo filter function $f(t)$.

Performance evaluation and optimization can now proceed much as in the case of open-loop wavefront reconstruction considered above. The expected residual phase variance $\left\langle\sigma^{2}\left(R s_{f}\right)\right\rangle$ is evaluated using Eq. (7) with $a=R s_{f}$. The covariance matrix $\left\langle\left(a_{*}-R s_{f}\right)\left(a_{*}-R s_{f}\right)^{T}\right\rangle$ is computed using the equivalent of Eq. (10) with $s_{f}$ substituted for each appearance of the open-loop measurement $s_{0}$. The covariance matrices $\left\langle s_{f} s_{f}^{T}\right\rangle$ and $\left\langle a_{*} s_{f}^{T}\right\rangle$ are in turn evaluated in terms of the statistics of $x_{f}, x$, and $n_{f}$.

For numerical computations, of course, the covariances involving the temporally filtered turbulence profiles $x_{f}$ may be more difficult to evaluate than the statistics of the instantaneous profiles $x$. The of validity of the result also depends strongly upon constraint $R G_{a}=I$, a condition which holds for the standard least squares reconstructors $R_{L S}=\left(G_{a}^{T} G_{a}\right)^{-1} G_{a}^{T}$ and $R_{W L S}=\left(G_{a}^{T}\left\langle n n^{T}\right\rangle^{-1} G_{a}\right)^{-1} G_{a}^{T}\left\langle n n^{T}\right\rangle^{-1}$ actually used in many AO systems. It does generally not hold for the minimum variance reconstructor $R_{*}$ defined by Eq. (11), but is is possible to determine the reconstructor $R_{c}$ that optimizes closed-loop performance subject to the condition $R G_{a}=I$. The result is the expression ${ }^{19}$

$$
R_{c} \equiv \arg \min \left\{\left\langle\sigma^{2}\left(R s_{f}\right)\right\rangle: R G_{a}=I\right\}=R_{*}-\left(I-R_{*} G_{a}\right)\left(G_{a}^{T}\left\langle s_{f} s_{f}^{T}\right\rangle^{-1} G_{a}\right)^{-1} G_{a}^{T}\left\langle s_{f} s_{f}^{T}\right\rangle^{-1} .
$$




\subsection{Servo Filter Optimization; Modal and Zonal Control}

Further optimization of the residual closed-loop phase variance $\left\langle\sigma^{2}\left(R s_{f}\right)\right\rangle$ is accomplished by tuning the temporal filter $f(t)$. This is essentially a matter of balancing the magnitudes of the reconstruction errors due to (i) the noise $n_{f}$ remaining after filtering and (ii) the latency and loss of fidelity in the filtered turbulence profile $x_{f}$. The value of $\left\langle\sigma^{2}\left(R s_{f}\right)\right\rangle$ is definitely a nonlinear function of the choice of filter $f$, but for classical AO control loops $f$ is defined by just one or several servo parameters. The prescription $f(t)=2 \pi f_{c}$ for all $t \geq 0$, for example, defines a 1-parameter family of type I control laws with the corresponding closed-loop impulse functions $f_{I R}(t)=2 \pi f_{c} \exp \left(-2 \pi f_{c} t\right)$. Minimizing $\left\langle\sigma^{2}\left(R s_{f}\right)\right\rangle$ over a parameterized family of temporal filters may be accomplished by applying a standard iterative algorithm for optimizing nonlinear functions, for example Newton's method or steepest descent. ${ }^{21}$

A basic limitation of this approach (and the AO control loop model formulated in subsection 2.3 above) is that all the components, or modes, of the phase disturbance must be corrected using a common value for $f$. In many cases, better results may be achieved by recognizing that each of these components is characterized by a distinct temporal power spectrum, and that the AO control algorithm can be tuned accordingly. Modal control $^{22}$ and regularized zonal control ${ }^{23}$ are two standard approaches, although space limitations prevent us from formulating them here.

\section{SPATIAL FREQUENCY DOMAIN REPRESENTATIONS}

The general results outlined above cannot actually be applied to a particular modeling problem until numerical values or analytical expressions are available for all of the terms describing the AO system and the observing scenario. These terms are the (i) wavefront propagation, sensing, and correcting operators, (ii) the phase variance metric $W_{\phi}$ and the related matrices $W_{a}, W_{x}$, and $C_{a x}$, and (iii) the covariance matrices describing the secondorder statistics of atmospheric turbulence and WFS measurement noise. We briefly summarize spatial frequency domain formulas for these quantities in the following subsections, using the usual geometrical optics models as starting points. All of these matrix- or operator-valued expressions are diagonal with respect to spatial frequency, so that AO performance may be evaluated or optimized by using the results developed in Section 2 by solving many small problems individually for each Fourier component.

\subsection{Wavefront propagation, sensing, and correction}

The abstract vectors $x, \phi, a$, and $s$ must now be defined more explicitly. In the spatial domain, the turbulence vector $x$ becomes a series of screens $x(r ; j)$, where $r$ is a two-dimensional coordinate in the plane orthogonal to the line-of-sight, and screen number $j$ is at range $h_{j}$ from the telescope. The phase vector $\phi$ becomes the function $\phi(r ; \theta)$, where $r$ represents coordinates in the telescope aperture plane and $\theta$ is a (continuous or discrete) parameter indicating the direction of a science object. The DM actuator command vector $a$ is replaced by arrays of commands $a\left(\Delta_{k} m ; k\right)$, where $\Delta_{k}$ is the inter-actuator spacing on DM number $k$ (imaged onto the telescope aperture plane), $m=\left(m_{x}, m_{y}\right)$ is a two-dimensional index ranging over the grid of actuators, and DM number $k$ is optically conjugate to a range $h_{k}^{\prime}$ from the telescope. Similarly, the WFS measurement vector $s$ becomes the two-dimensional function $s(\Delta n ; l)$, where $\Delta$ is the width of a sub-aperture in the aperture plane, $n=\left(n_{x}, n_{y}\right)$ is a two-dimensional index ranging over the grid of sub-apertures, and WFS number $l$ observes guidestar number $l$ in direction $\vartheta_{l}$ through the atmospheric turbulence layers and deformable mirrors. We note that (i) the subaperture width $\Delta$ is identical for all guidestars, (ii) all guidestars are at infinite range, and that (iii) all wavefront sensor pupils are conjugate to the telescope aperture plane.

The coordinates $r, n$, and $m$ are unbounded by any finite aperture, a key assumption that allows the processes of wavefront propagation, sensing, and correction to be represented as spatial filtering operations. The spatial Fourier transform of $x(r ; j)$ with respect to $r$ will be denoted as $\hat{x}(\kappa ; j)$, and similarly for the remaining functions. The transforms of $a\left(\Delta_{k} m ; k\right)$ and $s(\Delta n ; l)$ are periodic on square cells of width $1 / \Delta_{k}$ and $1 / \Delta$, respectively, since the DM actuator commands and WFS measurements are discrete functions of $m$ and $n$.

We now describe the spatial- and frequency domain representations of the wavefront operators introduced in Section 2 above, beginning with the turbulence-to-phase propagation operator $H_{x}$. Using the usual geometrical 
optics model, the effect of turbulence upon a plane wavefront propagating from a science object in direction $\theta$ is described by

$$
\phi(r ; \theta)=\sum_{j} x\left(r+h_{j} \theta ; j\right),
$$

since turbulence screen number $j$ is located at range $h_{j}$. Applying the Fourier shift theorem yields the result

$$
\hat{\phi}(\kappa, \theta)=\sum_{j} \exp \left(2 \pi i h_{j} \theta \cdot \kappa\right) \hat{x}(\kappa ; j) .
$$

The coefficients of the operator $H_{x}$ are just the complex exponentials appearing in the above equation, and it is clear that this is a sum of spatial filtering operations applied to each turbulence layer.

The remaining sensing and correcting processes $G_{x}, H_{x}$, and $H_{a}$ are compositions of multiple operators. All three of these quantities can be expressed as products of (i) a term describing the propagation of a wavefront through a series of screens (either turbulence layers or deformable mirror surfaces), together with either (ii) an operator $\mathcal{H}$ that transforms the DM actuator command vectors $a\left(\Delta_{k} m ; k\right)$ into the corresponding set of DM surface profiles $\alpha(r ; k)$, and/or (iii) an operator $\mathcal{G}$ that converts a guidestar wavefront $\varphi(r ; l)$ at the entrance pupil of a wavefront sensor into the resulting WFS measurement vector $s(\Delta n ; l)$. More specifically, we have $G_{x}=\mathcal{G} G_{x}^{\prime}, H_{a}=H_{a}^{\prime} \mathcal{H}$, and $G_{a}=\mathcal{G} G_{a}^{\prime} \mathcal{H}$, where the primed versions of the three operators are analogous to $H_{x}$ above, although the ranges to the layers and the directions of the wavefront propagations will generally be different.

It remains to formulate $\mathcal{H}$ and $\mathcal{G}$ and confirm that they are spatial filtering operations. The operator $\mathcal{H}$ is based on the usual model that represents the DM figure $\alpha(r ; k)$ as a linear superposition of the individual actuator influence functions, namely

$$
\alpha(r ; k)=\sum_{m} a\left(\Delta_{k} m ; k\right) h_{k}\left(r-\Delta_{k} m\right),
$$

where the index $m=\left(m_{x}, m_{y}\right)$ varies over all pairs of actuator indices, and $h_{k}(r)$ is the common influence function for all of the actuators on DM number $k$. If we restrict ourselves to the special case $h_{k}(x, y)=$ $\left[\sin \left(\pi \Delta_{k} x\right) /\left(\pi \Delta_{k} x\right)\right]\left[\sin \left(\pi \Delta_{k} y\right) /\left(\pi \Delta_{k} y\right)\right]$, the spatial Fourier transform of $\alpha$ may be written as

$$
\hat{\alpha}(\kappa ; k)= \begin{cases}\hat{a}(\kappa ; k) & \text { if } \max \left\{\left|\kappa_{x}\right|,\left|\kappa_{y}\right|\right\}<1 /\left(2 \Delta_{k}\right), \\ 0 & \text { otherwise, }\end{cases}
$$

and for this influence function the operator $\mathcal{H}$ is just a low-pass spatial filter.

The last quantity to be considered is the operator $\mathcal{G}$ mapping guidestar wavefronts $\varphi(r ; l)$ into WFS measurements $s(\Delta n ; l)$. Using the standard linear model for a wavefront gradient sensor, the measurement $s$ may be written in the form

$$
s(\Delta m ; l)=\Delta^{-2} \int d^{2} r \nabla \varphi(r ; l) w(r-\Delta m)+n(\Delta m ; l),
$$

where the two-dimensional integer index $m$ varies over all sub-aperture locations, the function $n$ represents the electronic noise in the measurement due to photon statistics, detector read noise, and background photons, and the "sub-aperture influence function" $w(r)$ is equal to unity for $r$ within a square sub-aperture of width $\Delta$, and is zero otherwise. Applying the convolution and differentiation theorems yields the result

$$
\begin{aligned}
\hat{s}(\kappa, l) & =2 \pi i \sum_{m}\left(\kappa+\Delta^{-1} m\right) \hat{\varphi}\left(\kappa+\Delta^{-1} m ; l\right) \hat{w}^{*}\left(\kappa+\Delta^{-1} m\right)+\hat{n}(\kappa ; l) \\
& =2 \pi i \kappa \hat{\varphi}(\kappa ; l) \hat{w}^{*}(\kappa)+\left[2 \pi i \sum_{m \neq 0}\left(\kappa+\Delta^{-1} m\right) \hat{\varphi}\left(\kappa+\Delta^{-1} m ; l\right) \hat{w}^{*}\left(\kappa+\Delta^{-1} m\right)+\hat{n}(\kappa ; l)\right] .
\end{aligned}
$$

¿From this last expression, it is clear that the wavefront sensing operator $\mathcal{G}$ is not purely a spatial filter. However, it behaves as a spatial filter on the component of $\hat{\varphi}$ defined within the passband $\max \left\{\left|\kappa_{x}\right|,\left|\kappa_{y}\right|\right\}<1 /(2 \Delta)$, with the term inside square brackets acting as a type of independent measurement noise. This measurement error is the sum of the WFS electronic noise proper, plus "aliasing" terms due to the higher frequency components of the phase profile $\varphi$. 


\subsection{Phase variance metric}

We define a special case of the phase variance metric $W_{\phi}$ introduced in Section 2 above by the formula

$$
\phi^{T} W_{\phi} \psi=\int d \theta W_{\Omega}(\theta) \int d \kappa\left[1-\left|\hat{W}_{A}(\kappa)\right|^{2}\right] \hat{\phi}^{*}(\kappa ; \theta) \hat{\psi}(\kappa ; \theta) .
$$

Here $\phi(r, \theta)$ and $\psi(r, \theta)$ are any two phase profiles in the telescope aperture plane for each direction $\theta$ within a field-of-view (FOV) $\Omega, W_{\Omega}$ is a non-negative FOV-weighting function normalized such that $\int d \theta W_{\Omega}(\theta)=1$, and $W_{A}$ is a non-negative telescope aperture function that has been similarly normalized to unity. Typical values for $W_{\Omega}$ include disks, squares, sums of discrete $\delta$-functions, and perhaps Gaussian profiles. The most common choice of $W_{A}$ is a disk of diameter $D$, in which case

$$
\hat{W}_{A}(\kappa)=2 J_{1}(\pi D \kappa) /(\pi D \kappa),
$$

where $J_{1}$ is a Bessel function of the first kind.

This choice of phase metric is diagonal with respect to spatial frequency by definition. It has been chosen because it may be shown that if $\hat{\phi}(\kappa ; \theta)$ is a random phase profile which is $\delta$-correlated with respect to $\kappa$, then

$$
\left\langle\phi^{T} W_{\phi} \phi\right\rangle=\int d \theta W_{\Omega}(\theta)\left\langle\int d r W_{A}(r)\left[\phi(r ; \theta)-\int d r^{\prime} W_{A}\left(r^{\prime}\right) \phi\left(r^{\prime} ; \theta\right)\right]^{2}\right\rangle
$$

In other words, the expected value of the metric is simply the expected value of the field- and aperture-averaged, piston-removed variance of $\phi$. We note that this result only holds for expected values, not for the individual realizations of the phase profile.

\subsection{Turbulence and noise statistics}

The atmospheric turbulence spectrum $\hat{x}(\kappa ; j)$ is $\delta$-correlated with respect to $\kappa$ and the layer index $j$. For a Von Karman power spectrum, its variance may be written in the form

$$
\left\langle|\hat{x}(\kappa ; j)|^{2}\right\rangle=c_{x} r_{0}^{5 / 3} C_{n}^{2}\left(h_{j}\right) \Psi(\kappa) / \sum_{k} C_{n}^{2}(k),
$$

where $c_{x}=2.29 \times 10^{-2}$ on account of the factors of $2 \pi$ appearing in our choice of convention for the Fourier transform, $r_{0}$ is the turbulence-induced effective coherence diameter, $C_{n}^{2}\left(h_{j}\right)$ is the (discretized) refractive index structure constant for the atmospheric layer at range $h_{j}$, and $\Psi(\kappa)=\left(\kappa^{2}+L_{0}^{-2}\right)^{-11 / 6}$ is the Von Karman spectrum with an outer scale of $L_{0}$.

The WFS measurement noise $n(\Delta m ; l)$ is statistically independent for distinct guidestars, sub-aperture locations, and $(x-$ or $y-)$ components. The noise variance is identical for all measurements from a given guidestar, with a value denoted as $\sigma_{n}^{2}(l)$ for guidestar number $l$. We recall that the variable $n$ denotes only the electronic component of the WFS measurement noise, and that the total noise includes a contribution from spatial aliasing according to Eq. (21) above. This additional noise source will be partially correlated between sub-apertures and guidestars according to the statistics of the turbulence $\hat{x}$ and the specific wavefront sensing geometry.

According to section 2.3, we also require formulas for the second-order statistics of $x_{f}$, and $n_{f}$ to evaluate the dynamic performance of a closed-loop AO system. Here $x_{f}$ and $n_{f}$ are obtained by convolving $x$ and $n$ with the the servo impulse response function $f_{I R}$ corresponding to the servo filter function $f$. A Fourier domain representation for the filtered turbulence profile $x_{f}$ can be derived by assuming that $x$ evolves according to the Taylor hypothesis,

$$
x(r ; j, t)=x\left(r-t v_{j} ; j, 0\right),
$$

where $v_{j}$ is the wind velocity of atmospheric layer number $j$. Using the shift theorem and the definition of the impulse response function $f_{I R}$, it may be shown that $\hat{x}_{f}(\kappa ; j, t)$ may be expressed as

$$
\hat{x}_{f}(\kappa ; j, t)=\exp \left(2 \pi i t v_{j} \cdot \kappa\right)\left[\frac{\hat{f}\left(v_{j} \cdot \kappa\right)}{1+\hat{f}\left(v_{j} \cdot \kappa\right)}\right] \hat{x}(\kappa ; j, 0),
$$


The expected values of $x_{f} x_{f}^{T}$ and $x x_{f}^{T}$ may now be computed using this result, Eq. (25) for the statistics of the instantaneous profile $x$, and the values of the wind velocities $v_{j}$. For example, with a known windspeed, an unknown and uniformly distributed wind direction, and the type I servo filter describe in Section 2.4 above we obtain

$$
\left\langle\left|\hat{x}_{f}(\kappa ; j)\right|^{2}\right\rangle=\left\langle\hat{x}(\kappa ; j) \hat{x}_{f}^{*}(\kappa ; j)\right\rangle=\frac{\left\langle|\hat{x}(\kappa ; j)|^{2}\right\rangle}{\left(1+\left|v_{j} \kappa / f_{c}\right|^{2}\right)^{1 / 2}} .
$$

Finally, the second-order statistics of the temporally filtered noise $n_{f}$ is easily computed from the definition of the impulse response function $f_{I R}$ and the assumption that the noise is temporally uncorrelated.

\section{COMPARISONS AGAINST CLASSICAL SCALING LAWS FOR NGS AO}

The expressions for operators, performance metrics, and covariance matrices derived above may now be combined with the linear systems models discussed in Section 2 to evaluate the performance of a variety of AO systems concepts. In the general case these evaluations must be performed numerically, but for classical natural guide star (NGS) AO systems it is possible to derive simplified formulas for the individual impacts of a variety of AO error sources considered separately. These error sources include (i) DM fitting error, (ii) WFS spatial aliasing, (iii) WFS measurement noise, (iv) anisoplanatism, and (v) servo lag. In this section we, briefly summarize the case of WFS measurement noise, where the deviation between the results of frequency domain model and more detailed Monte Carlo simulations that model aperture edge effects is the greatest.

The so-called "noise gain" of the wavefront reconstruction algorithm is computed by neglecting atmospheric turbulence and evaluating the ratio between the mean-square reconstructed phase error and the mean-square WFS measurement noise. Using Eq.'s (7) and (10) with the results of Section 3, the noise gain for the classical least-squares reconstructor may be written in the form

$$
\frac{\left\langle\sigma^{2}\left(R_{L S} s_{0}\right)\right\rangle}{\sigma_{n}^{2}}=\frac{1}{4 \pi^{2}} \int_{-1 / 2}^{1 / 2} \int_{-1 / 2}^{1 / 2} \frac{d \kappa}{\kappa^{2}}\left\{1-\left[\frac{2 J_{1}(\pi \kappa D / \Delta)}{(\pi \kappa D / \Delta)}\right]^{2}\right\} \frac{1}{\left[\operatorname{sinc}\left(\pi \kappa_{x}\right) \operatorname{sinc}\left(\pi \kappa_{y}\right)\right]^{2}} .
$$

The reconstructor noise gain is a function of the order of correction $D / \Delta$, where $\Delta$ is equal to both the width of the subaperture and the DM interactuator spacing.

Fig. 1 plots the values obtained from Eq. (29), together with the curve fit $0.150+0.083 \log \left[(\pi / 4)(D / \Delta)^{2}\right]$. The logarithmic dependence upon the order of the AO system arises from the term $\kappa^{-2}$ appearing in the integrand in Eq. (29). This functional form matches a variety of results obtained by more detailed analyses in the spatial domain, ${ }^{19}$ although the numerical value is too small by a factor of about 17 per cent in the limit of very large $D / \Delta$. We attribute this optimism to the fact that the spatial frequency domain model utilizes WFS measurements on a grid of sub-aperture locations that extends outside of the physical boundary of the evaluation aperture. Measurements from these phantom sub-apertures reduce the effect of WFS measurement noise on the reconstruction of low-order phase distortions.

\section{COMPARISONS AGAINST MONTE CARLO SIMULATIONS}

We have tested the accuracy of the frequency domain model for more complex AO configurations by evaluating 8-meter class multi-conjugate $\mathrm{AO}(\mathrm{MCAO})$ and ground-layer $\mathrm{AO}$ (GLAO) systems, and comparing the results obtained against Monte Carlo simulation codes that have been previously described. ${ }^{3,24}$ The basic models for wavefront propagation, sensing, and correction used in these simulations are essentially as described in Section 3 above, so that these comparisons highlight the impact of neglecting aperture edge effects in the spatial frequency domain model. The level of agreement obtained ranges from good to excellent in all cases considered to date. Sample results for MCAO are described in the following paragraphs.

This sample case is based very loosely upon the MCAO design now being fabricated for the 8-meter Gemini South telescope at Cerro Pachon, Chile. ${ }^{25}$ The simulated atmospheric profile included 6 layers at altitudes of $0,2,4,8,12$, and 16 kilometers, with relative $C_{n}^{2}(h)$ strengths of $0.6,0.2,0.05,0.05,0.07$, and 0.03 . The strengths of the layers were scaled to obtain an effective coherence diameter $r_{0}$ of 0.15 meters at a wavelength of $0.5 \mu \mathrm{m}$, with an isoplanatic angle $\theta_{0}$ of 2.41 arc seconds. The six layers had velocities of $5,7,10,15,25$, and 15 


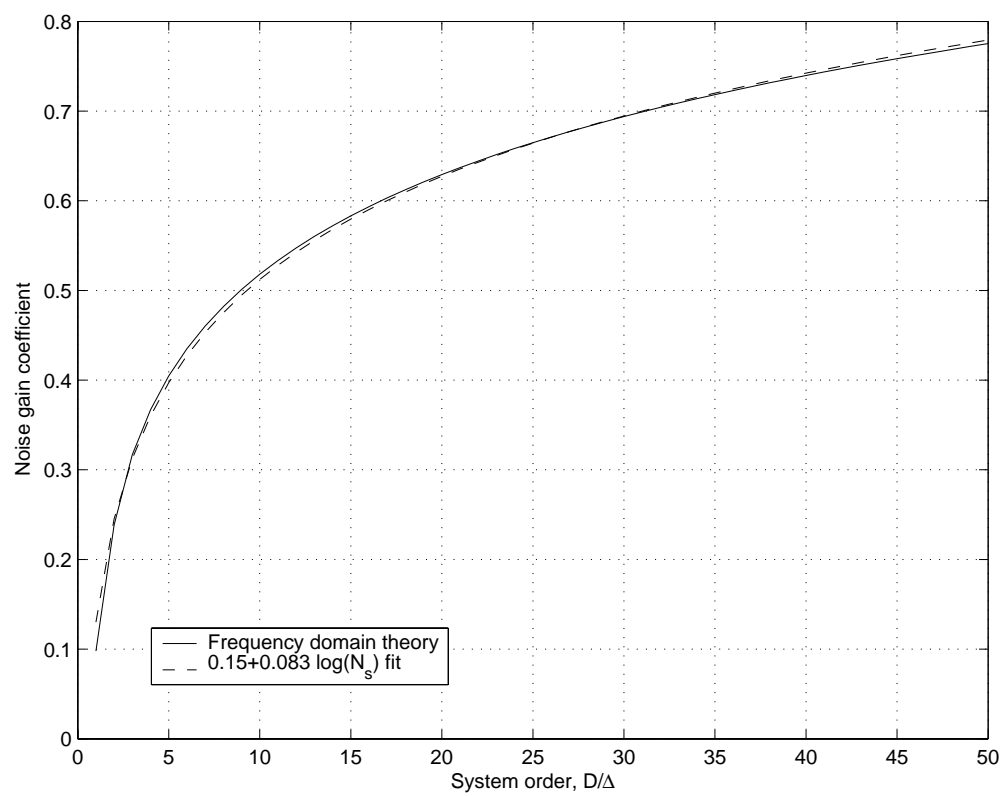

Figure 1. Wavefront reconstruction noise gain for NGS AO

This figure plots the noise gain coefficient (computed according to Eq. (29) above) for a least squares wavefront reconstruction algorithm as a function of the order of the AO system. The dashed line plots the value of a simplified scaling law that provides a good fit the numerical results.

$\mathrm{m} / \mathrm{sec}$, yielding a Greenwood frequency $f_{g}$ of $25 \mathrm{~Hz}$. The outer scale $L_{0}$ was equal to infinity for all turbulence layers. The Monte Carlo simulations used periodic turbulence screens with $2048^{2}$ points, a grid spacing of $1 / 32$ meters, and a width of 64 meters. The impact of the pseudo inner- and outer-scales induced by these sampling parameters has not been investigated.

The telescope aperture diameter was $8 \mathrm{~m}$, with no central obscuration. MCAO performance was optimized and evaluated over a one arc minute square, sampled discretely at the nine points with coordinates $([-30,0,30],[-30,0,30])$ sec (arc seconds) in the spatial domain simulation. Five natural guide star were located in the directions $(0,0)$ and $( \pm 30, \pm 30)$ sec, with a WFS sub-aperture width of $0.5 \mathrm{~m}$. Partially illuminated edge sub-apertures were retained in the WFS measurement vector for the Monte Carlo simulation. Three deformable mirrors were located at conjugate ranges of 0,5 , and 10 kilometers, with inter-actuator spacings of $0.5,0.5$, and $1.0 \mathrm{~m}$. The Monte Carlo simulation used a linear spline influence function, in contrast with the $\sin (\pi \Delta x) /(\pi \Delta x)$ influence function introduced in Section 3 above.

AO system performance was evaluated with a minimum variance wavefront reconstruction algorithm. Closedloop performance was evaluated for a $12 \mathrm{~Hz}$ control bandwidth, and the impact of WFS measurement noise was evaluate for a sub-aperture noise equivalent angle of 0.01 sec per $\mathrm{Hz}^{1 / 2}$. For the Monte Carlo simulation, the WFS sampling rate was $800 \mathrm{~Hz}$, and DM commands were updated using a simple integrator with $3 \mathrm{~dB}$ error rejection at $12 \mathrm{~Hz}$.

The performance predictions computed by the two models were compared by successively simulating problems of increasing complexity that incrementally added one or two error sources at a time. The problems considered were: (i) DM fitting and WFS spatial aliasing for a 8-meter class NGS AO system with a 0.5 meter sub-aperture and actuator pitch; (ii) the increased phase variance due to tomographic wavefront reconstruction and wide FOV wavefront compensation in a NGS MCAO system with no WFS measurement noise or servo lag; (iii) the servo lag error for a $12 \mathrm{~Hz}$ control bandwidth; and (iv) the error due to WFS noise for a noise equivalent angle (NEA) of 0.01 sec $/ \mathrm{Hz}^{1 / 2}$. An "error budget" for these four terms was created by differencing (in quadrature) successive performance estimates from each of the models. The parameters for this test case were selected so that roughly 
Table 1. Comparison of frequency domain performance estimates vs. the results of Monte Carlo simulations for a sample NGS MCAO system. The values are RMS wavefront errors in units of nm. See the text for descriptions of the AO system, the turbulence profile, and further definition of the error terms.

\begin{tabular}{|l|r|r|}
\hline Model & Frequency Domain & Monte Carlo \\
\hline Fitting error & 110.4 & 115.4 \\
Tomography & 89.7 & 92.5 \\
Servo lag & 89.8 & 88.4 \\
WFS noise & 83.7 & 94.4 \\
Overall & 188.9 & 196.5 \\
\hline
\end{tabular}

Table 2. Comparison of Strehl ratio estimates in J and K bands. These results correspond to the field-averaged RMS wavefront errors summarized in table 1. See the text for descriptions of the MCAO system and turbulence profile.

\begin{tabular}{|l|l|r|r|r|}
\hline Band & \multicolumn{1}{|c|}{ Model } & Center & Edge & Corner \\
\hline \multirow{2}{*}{$\mathrm{J}$} & Frequency Domain & 0.474 & 0.371 & 0.327 \\
\cline { 2 - 5 } & Monte Carlo & 0.442 & 0.357 & 0.337 \\
\hline \multirow{2}{*}{$\mathrm{K}$} & Frequency Domain & 0.786 & 0.726 & 0.696 \\
\cline { 2 - 5 } & Monte Carlo & 0.768 & 0.716 & 0.703 \\
\hline
\end{tabular}

equal contributions were obtained from each of the four error sources.

Table 1 summarizes the RMS wavefront error budgets computed using the two approaches. The overall performance estimates differ by about 4 per cent, with the individual terms in the error budget differing by from 2 to 10 per cent. The largest discrepancy occurs for WFS measurement noise; this is evidently the most significant error made by neglecting aperture edge effects in the spatial frequency domain model. The differences for the remaining terms range from 2 to 4 per cent.

Table 2 lists the long-exposure Strehl ratios computed via analysis and Monte Carlo simulation in the $\mathrm{J}$ and $\mathrm{K}$ spectral bands at the center, edge, and corner of the 1 arc minute square FOV. The agreement is again good to a few per cent (relative), and the analytical results provide a good estimate of the variations in performance across the field-of-view. Finally, Fig. 2 is a (log-stretch) plot of the $\mathrm{J}$ and $\mathrm{K}$ band PSFs computed at the corner of the FOV using the two techniques. It may be seen that (i) the diameters of the seeing-limited halos are in agreement, (ii) the diameters of the central cores are in agreement, and (iii) the shape and size of the radial asymmetries may be in agreement, althought more simulation trials will be required to confirm or reject this preliminary assertion. [We hope to have these simulations completed in time for the proceedings.]

\section{ACKNOWLEDGMENTS}

The author presents this paper as part of the work of the Thirty Meter Telescope (TMT) Project. TMT is a partnership of the Association of Universities for Research in Astronomy (AURA), the Association of Canadian Universities for Research in Astronomy (ACURA), the California Institute of Technology and the University of California. The partners gratefully acknowledge the support of the Gordon and Betty Moore Foundation, the US National Science Foundation, the National Research Council of Canada, the Natural Sciences and Engineering Research Council of Canada, and the Gemini Partnership.

The New Initiatives Office is a partnership between two divisions of the Association of Universities for Research in Astronomy (AURA), Inc.: the National Optical Astronomy Observatory (NOAO) and the Gemini Observatory.

NOAO is operated by AURA under cooperative agreement with the National Science Foundation (NSF). 


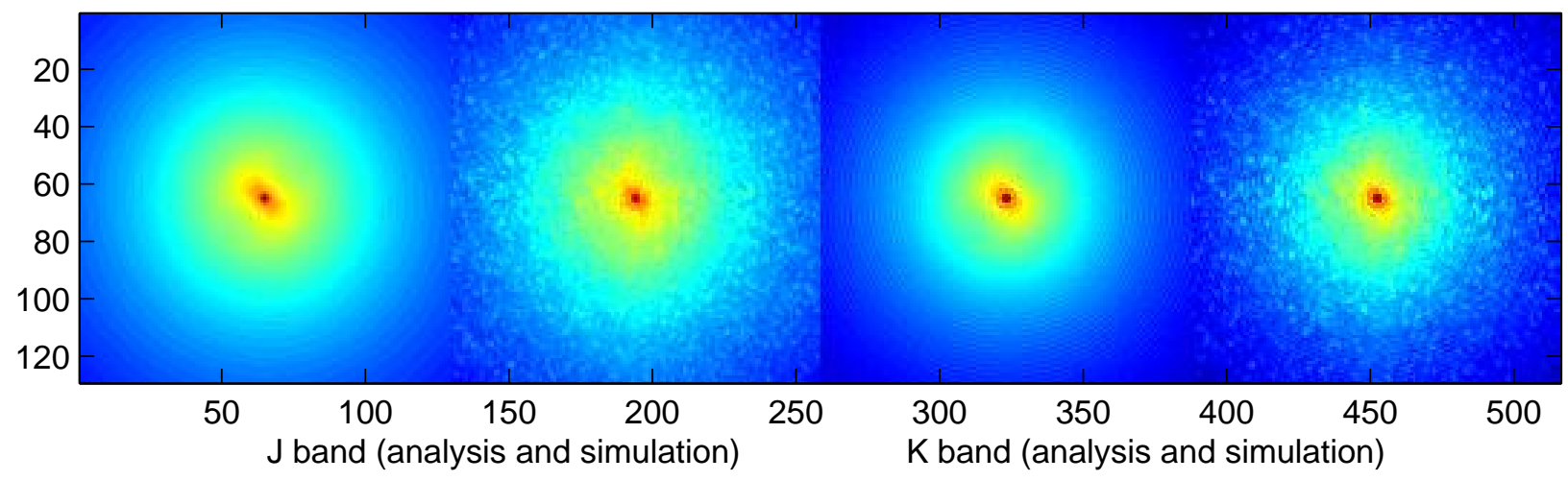

Figure 2. PSF estimates at the corner of the MCAO field-of-view

This figure is a log stretch of the $\mathrm{J}$ and $\mathrm{K}$ band point spread functions computed via frequency domain analysis and Monte Carlo simulation for the MCAO system and atmospheric turbulence parameters described in the text. The images are Nyquist sampled with a width equal to 64 diffraction-limited blur diameters. The simulated PSFs are averages of 500 instantaneous values collected at a sampling rate of $800 \mathrm{~Hz}$.

The Gemini Observatory is operated by AURA under a cooperative agreement with the NSF on behalf of the Gemini partnership: the National Science Foundation (United States), the Particle Physics and Astronomy Research Council (United Kingdom), the National Research Council (Canada), CONICYT (Chile), the Australian Research Council (Australia), CNPq (Brazil) and CONICET (Argentina).

\section{REFERENCES}

1. Adaptive Optical System Technologies II, P. L. Wizinowich and D. Bonaccini, eds., Proc. Soc. Photo-Opt. Instrum. Eng. 4839 (2003).

2. Extremely Large Telescopes II, T. Andersen and A. Ardeberg, eds., Proc. Soc. Photo-Opt. Instrum. Eng. (in press).

3. B. L. Ellerbroek, L. Gilles, and C. R. Vogel, "Numerical simulations of multiconjugate adaptive optics wave-front reconstructors on giant telescopes," Appl. Opt. 42, 4811-4818 (2003).

4. L. Gilles, B. L. Ellerbroek, and C. R. Vogel, "Preconditioned conjugate gradient wavefront reconstructors for multi-conjugate adaptive optics," Appl. Opt. 42, 5233-5250 (2003).

5. B. L. Ellerbroek and C. R. Vogel, "Simulations of closed-loop wavefront reconstruction for multi-conjugate adaptive optics on giant telescopes," in Astronomical Adaptive Optics Systems and Applications, R. K. Tyson and M. Lloyd-Hart, eds., Proc. Soc. Photo-Opt. Instrum. Eng. 5169, 206-217 (2003).

6. A. J. Ahmadia and B. L. Ellerbroek, "Parallelized simulation code for multiconjugate adaptive optics," in Astronomical Adaptive Optics Systems and Applications, R. K. Tyson and M. Lloyd-Hart, eds., Proc. Soc. Photo-Opt. Instrum. Eng. 5169, 218-227 (2003).

7. C. Arcidiacono, E. Diolaiti, R. Ragazzoni, A. Baruffolo, A. Brindisi, J. Farinato, and E. Vernet-Viard, "Sky coverage and Strehl ratio uniformity in layer-oriented MCAO systems," in Astronomical Adaptive Optics Systems and Applications, R. K. Tyson and M. Lloyd-Hart, eds., Proc. Soc. Photo-Opt. Instrum. Eng. 5169, 169-180 (2003).

8. D. L. Fried, "Anisoplanatism in adaptive optics," J. Opt. Soc. Am. 72, 52 (1982).

9. D. P. Greenwood, "Bandwidth specifications for adaptive optics systems," J. Opt. Soc. Am. 67, 390-392 (1977).

10. G. A. Tyler, "Turbulence-induced adaptive-optics performance evaluation: degradation in the time domain," J. Opt. Soc. Am. A 1, 251-262 (1984).

11. L. A. Poyneer and B. Macintosh, "Spatially filtered wave-front sensor for high-order adaptive optics," J. Opt. Soc. Am. A 21, 810-819 (2004). 
12. A. Tokovinin and M. Le Louarn, "Isoplanatism in a multiconjguate adaptive optics system," J. Opt. Soc. Am. A 17, 1819-1827 (2000).

13. A. Tokovinin and E. Viard, "Limiting precision of tomographic phase estimation," J. Opt. Soc. Am. A 18, $873-8827$ (2001).

14. F. Rigaut, J.-P. Veran, and O. Lai, "An analytical model for Shack-Hartmann based adaptive optics systems," Adaptive Optical Systems Technologies, D. Bonaccini and R. K. Tyson, eds., Proc. Soc. PhotoOpt. Instrum. Eng. 3353, 1038-1048 (1998).

15. L. Jollisaint and J.-P. Veran, "Fast computation and morphologic interpretation of the Adaptive optics point spread function," in Beyond Conventional Adaptive Optics, R. Ragazzoni, N. Hubin, S. Esposito, and E. Vernet, eds., ESO Conf. and Work. Proc. 58, 201-208 (2002).

16. E. P. Wallner, "Optimal wave-front reconstruction for compensated imaging," J. Opt. Soc. Am. 73, 17711776 (1983).

17. B. M. Welsh and C. S. Gardner, "Effects of turbulence-induced anisoplanatism on the imaging performance of adaptive-astronomical telescopes using laser guide stars," J. Opt. Soc. Am. A 8, 69-80 (1991).

18. D. C. Johnston and B. M. Welsh, "Analysis of multiconjugate adaptive optics," J. Opt. Soc. Am. A 11, 394-408 (1994).

19. B. L. Ellerbroek, "First-order performance evaluation of adaptive-optics systems for atmospheric-turbulence compensation in extended-field-of-view astronomical telescopes," J. Opt. Soc. Am. A 11, $783-805$ (1994).

20. T. Fusco, J. M. Conan, G. Rousset, L. M. Mugnier, and V. Michau, "Optimal wave-front reconstruction strategies for multi-conjugate adaptive optics," J. Opt. Soc. Am. A 18, 2527-2538 (2001).

21. W. H. Press, B. P. Flanner, S. A. Teukolosky, and W. T. Vetterling, Numerical Recipes, (Cambridge U. Press, Cambridge, 1987).

22. C, Boyer, E. Gendron, and P. Y. Madec, "Adaptive optics for high resolution imagery: control algorithms for optimized modal corrections," in Lens and Optical Systems Design, H. Zuegge, eds., Proc. Soc. PhotoOpt. Instrum. Eng. 1780, 943-957 (1992).

23. B. L. Ellerbroek, "Optimizing closed-loop adaptive-optics performance with use of multiple control bandwidths," J. Opt. Soc. Am. A 11, 2871-2886 (1994).

24. M. Le Louarn, C. Verinaud, J. Braud, E. Fedrigo, and V. Korkiakoswki, "Simulations of (MC)AO for a 100-m telescope, Advancements in Adaptive Optics, D. Bonaccini, B. L. Ellerbroek, and R. Ragazzoni, eds., Proc. Soc. Photo-Opt. Instrum. Eng. 5490 (in press).

25. On-line documentation for the Gemini-South MCAO Preliminary Design Review, http://www.gemini.edu . 